\title{
Using Electronic Drug Monitor Feedback to Improve Adherence to Antiretroviral Therapy Among HIV-Positive Patients in China
}

\author{
Lora L. Sabin · Mary Bachman DeSilva • \\ Davidson H. Hamer · Keyi Xu · Jianbo Zhang • \\ Tao Li · Ira B. Wilson · Christopher J. Gill
}

Published online: 22 September 2009

(C) The Author(s) 2009. This article is published with open access at Springerlink.com

\begin{abstract}
Effective antiretroviral therapy (ART) requires excellent adherence. Little is known about how to improve ART adherence in many HIV/AIDS-affected countries, including China. We therefore assessed an adherence intervention among HIV-positive patients in southwestern China. Eighty subjects were enrolled and monitored for 6 months. Sixty-eight remaining subjects were randomized to intervention/control arms. In months 7-12, intervention
\end{abstract}

L. L. Sabin · M. B. DeSilva · D. H. Hamer

Center for International Health and Development,

Boston University, Boston, MA, USA

L. L. Sabin $(\bowtie) \cdot$ M. B. DeSilva · D. H. Hamer

Department of International Health, Center for International

Health and Development, Boston University School of Public

Health, 801 Massachusetts Avenue, Crosstown Center,

3rd Floor, Boston, MA 02118, USA

e-mail: 1sabin@bu.edu

D. H. Hamer

Infectious Diseases Section, Department of Medicine,

Boston University School of Medicine, Boston, MA, USA

K. Xu

Department of STDs and Dermatology, Ditan Hospital,

Beijing, China

J. Zhang

Department of STDs and Dermatology,

Dali Second People's Hospital, Dali, China

T. $\mathrm{Li}$

Boston University School of Public Health, Boston, MA, USA

I. B. Wilson

Institute for Clinical Research and Health Policy Studies,

Tufts New England Medical Center, Boston, MA, USA

C. J. Gill

Novartis Vaccines, Cambridge, MA, USA subjects were counseled using EDM feedback; controls continued with standard of care. Among randomized subjects, mean adherence and CD4 count were 86.8 vs. $83.8 \%$ and 297 vs. 357 cells/ $\mu \mathrm{l}$ in intervention vs. control subjects, respectively. At month 12, among 64 subjects who completed the trial, mean adherence had risen significantly among intervention subjects to $96.5 \%$ but remained unchanged in controls. Mean CD4 count rose by 90 cells/ $\mu \mathrm{l}$ and declined by 9 cells $/ \mu$ l among intervention and control subjects, respectively. EDM feedback as a counseling tool appears promising for management of HIV and other chronic diseases.

Keywords AIDS - Medication adherence .

Antiretroviral medication - China .

Electronic drug monitoring

\section{Introduction}

Successful antiretroviral therapy (ART) requires HIVpositive patients to maintain high adherence to medication regimes [1-4]. Although recent research suggests that nonnucleoside reverse transcriptase inhibitor (NNRTI) based regimens are more forgiving of imperfect adherence [5], near perfect adherence that maximizes achievement of sustained viral suppression remains a central goal of therapy $[6,7]$. Poor adherence also has important societal consequences that extend beyond the individual. ART is cost effective [8-11] but not free, and its cost-effectiveness declines with reduced adherence, since the same expenditure yields declining benefits. Poor adherence also contributes to drug-resistant strains of HIV, which are not only associated with increased mortality in patients $[12,13]$ but also can be transmitted to others $[14,15]$. 
A number of interventions designed to improve ART adherence have been assessed in recent years. Two rigorous analyses of the efficacy of intervention trials conducted between 1996 and 2005 in high income countries [7, 16] both found a modest, but statistically significant, positive effect on adherence in intervention vs. control subjects when data from all the studies were pooled. This is encouraging because it suggests that interventions can beneficially affect adherence behaviors. However, adherence success varied substantially across the studies, with only about $25 \%$ of interventions efficacious. These and more recent studies indicate that many of the most successful interventions to date have involved providing adherence- and treatment-related information to patients and/or engaging them in interactive discussions of cognition, motivation, and expectations regarding adherence [17-20].

Electronic drug monitors (EDM) have been instrumental in monitoring ART adherence and in defining the relationship between adherence and HIV-related clinical outcomes [4, 21-23]. Two recent US-based studies also found a positive effect on ART adherence of multi-faceted interventions that included providing patients with their EDM data. One found that a self-management intervention implemented over 12 weeks increased the likelihood that patients would take $\geq 80 \%$ of their pills [24]. While this outcome is positive, $80 \%$ adherence is below the optimal level for long-term viral suppression. Rosen et al. similarly found that providing a contingency management intervention to HIV-positive patients with a history of substance abuse led to a significant increase in adherence, from $61 \%$ at baseline to $76 \%$ during the 16-week intervention phase, and a decline in HIV viral loads, though the differences in these outcomes were not statistically significant after 16 weeks of follow-up [25].

China is experiencing one of Asia's most serious HIV/ AIDS epidemics [26]. The most recent global and national reports indicate that approximately 700,000 Chinese were living with HIV/AIDS at the end of 2007 [27] and that new infections in China continue to increase steadily [28]. The majority of HIV-infected Chinese live in border provinces such as Yunnan, Guangxi, and Sichuan, where the virus has spread quickly due to a concurrent rise in injection drug use (IDU), and in central provinces including Henan and Anhui, where villagers were infected in the early-mid 1990s from contaminated blood collection practices [2931]. China has responded to its growing HIV/AIDS epidemic by rapidly expanding provision of free ART [3234]. By October 2007, approximately 32,000 individuals in 31 provinces across China were receiving ART [27]. A 2005 progress report on the ART scale-up noted poor adherence among farmers in the central provinces, blaming it for rising drug resistance in that region [35]. Yet, despite awareness of the importance of ART adherence, little is known about factors that influence adherence among Chinese patients or about potentially effective adherence interventions. As in most developing countries, the published literature is limited to descriptive and qualitative studies regarding barriers to adherence [36-38].

In the China Adherence for Life (AFL) study, we assessed the impact of systematically integrating EDM data into HIV clinical care - a process we term 'EDM feedback'-on ART adherence among HIV-positive individuals in southwestern China. Our hypothesis was that providing patients with counseling informed by objective data on their recent adherence behavior would enhance the effectiveness of adherence counseling, motivate patients to improve their medication-taking, and thereby improve ART adherence. We were implicitly testing the validity of an information-motivation-behavior skills model that has been used to describe the factors affecting adherence to ART [39]. This intervention was the final component of a three-part project that also described barriers to adherence [37] and, after measuring adherence using multiple methods over a 6-month period, identified the most accurate measure of adherence in this Chinese population [40]. The present paper presents the results of the intervention phase of the study. Our primary aim was to determine whether EDM feedback improved adherence 6 months after initiation of the intervention. A secondary aim was to assess whether EDM feedback improved HIV disease markers (CD4 count and viral suppression).

\section{Methods}

\section{Study Site and Subjects}

Adherence for Life was a non-blinded randomized controlled intervention trial conducted at the Dali Second People's Hospital (DSPH) in Dali, Yunnan province. Dali is home to approximately 40,000 residents, primarily of ethnic minority background. Over $80 \%$ of HIV-positive individuals in Yunnan are estimated to have been infected through IDU [41]. HIV-positive patients attending the DSPH Dermatology and Sexually Transmitted Diseases Clinic, where free ART has been provided since mid-2005, were enrolled. When enrollment began in June 2006, a total of 3 clinicians and 6 nurses were providing care and ART to approximately 70 patients. They all received twice-daily NNRTI regimens consisting of nevirapine or efavirenz plus lamivudine with stavudine or zidovudine, which were collected monthly at the DSPH.

Patients were eligible for participation if they were on ART and aged 18 years or above; there were no exclusion criteria. All eligible patients were approached by a 
clinician, who described the study and referred interested patients to a local study team member. The latter provided a detailed explanation of the study's purpose and requirements and obtained written informed consent prior to enrolling each patient. All subjects received a monthly stipend of 150 yuan (approximately US\$19) as compensation for lost work time and travel costs associated with study participation. The study was approved by the institutional review boards of Boston University and Ditan Hospital, Beijing.

\section{Sample Size}

Our target sample size of 80 was based on the number needed to detect a $15 \%$ improvement in mean adherence in the intervention arm, assuming a baseline adherence of $70 \%$, with $90 \%$ power and alpha 0.05 , and allowing for $10 \%$ attrition.

\section{Pre-Intervention Procedures}

In the pre-intervention period (months 1-6), we monitored subjects' adherence without providing the EDM data to them or their clinicians (Fig. 1). To do so, at enrollment each subject was given an EDM pill bottle $\left(\right.$ Med-ic ${ }^{\mathrm{TM}}$, Ottawa, Canada) for one of his/her ART medications. In most cases $(64 \%)$ this was nevirapine, because it fit most easily into the EDM bottle, although a minority of subjects' EDM bottles contained zidovudine or lamivudine. Subjects were informed about the monitoring aspect of the EDM bottles and, to ensure the collection of accurate data, were instructed to keep the medication (but only this one medication) in the bottle, not to play with it (opening/closing) or to take out more than one dose at a time, and to recap the bottle tightly after each use. Throughout the subsequent 12 months of data collection, when subjects made monthly clinic visits to refill their medications, a study team member downloaded their EDM data. Each subject also completed an adherence self-report that referred to medication-taking behavior during the previous week and month.

\section{Randomization}

In month 6 , subjects were stratified into high or low adherence groups based on whether they had optimal adherence during the pre-intervention period (defined as maintaining a mean of $\geq 95 \%$, as measured by EDM, throughout months 1-5). Subjects within each adherence group were then randomized to the intervention or control arm, ensuring that equal numbers of high and low adherers were allocated to each arm. This was performed on site, through a block randomization process as follows: at each subject's month 6 visit, the subject pulled an unmarked allocation envelope, the inside of which had a single paper stamped with either "intervention" or "control", from a larger envelope that had originally held ten such allocation envelopes, five for each arm. When each large envelope was empty, it was replaced with another large envelope, similarly containing ten allocation envelopes.
Fig. 1 Study profile. The study involved 12 months of data collection for each subject; with rolling admission from June to November 2006, Months 1-6 of data collection took place from June 2006 to May 2007 and Months 7-12 of data collection took place from January to November 2007
Enrollment:

June-November 2006

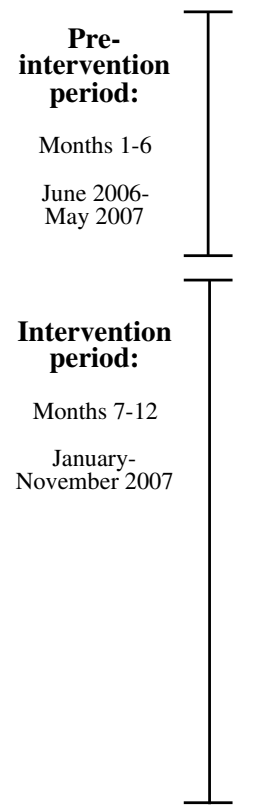

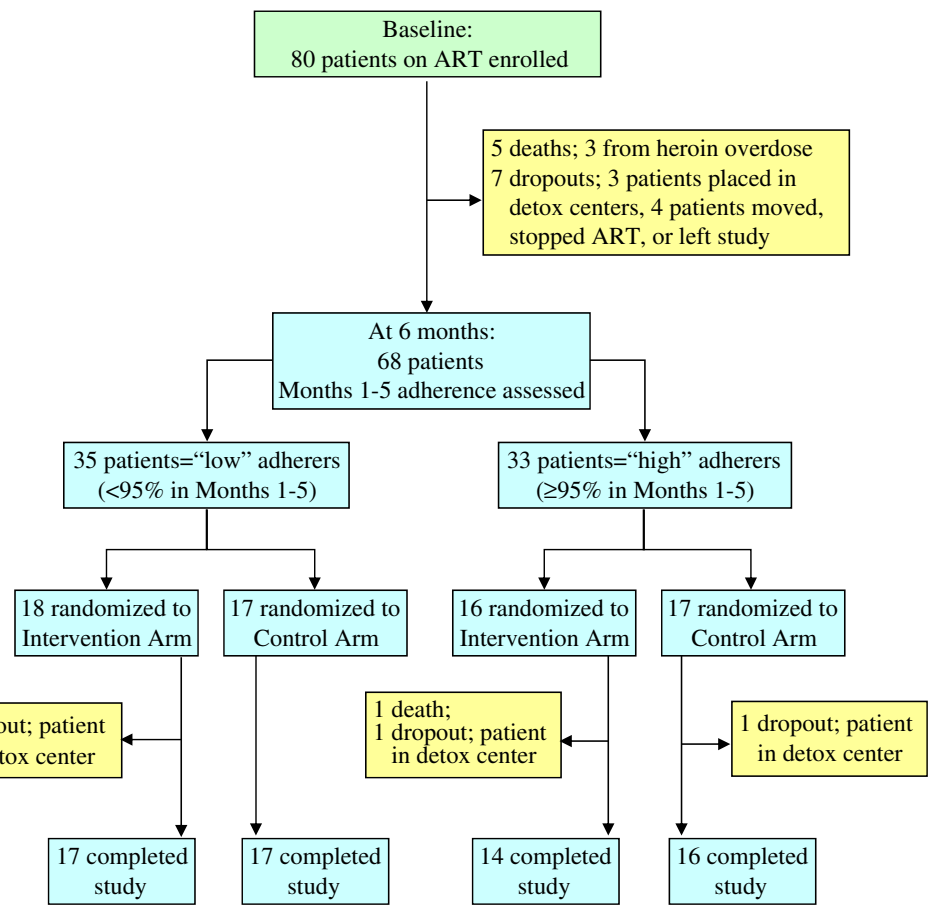


Intervention

In the intervention period (months 7-12), when intervention subjects came to the clinic for a monthly visit, a study team member downloaded and reviewed the subject's previous month's EDM data. Each subject found to be less than $95 \%$ adherent according to the EDM data was 'flagged' for counseling with a clinic physician or nurse utilizing the EDM information immediately following regular clinic visit activities. The data were provided to both the subject and his/her clinician as a printout summarizing the percent of doses taken, the percent of doses taken on time, and a visual display of doses taken by time. This process of flagging and counseling was specific to each clinic visit, such that if a subject was counseled in Month 8 but had EDM-measured adherence $\geq 95 \%$ at the Month 9 visit, no flagging for counseling occurred.

In each counseling session, the clinician reviewed the EDM printout with the subject, explored reasons for missed or off-time doses, and inquired about problems or challenges the subject might be having. Beyond this, counseling sessions did not follow a script. This was designed to accommodate each clinician's counseling style, allow for an individually-focused discussion of adherence behavior, and encourage each subject-clinician pair to devise personalized strategies to improving adherence. In this regard, the EDM feedback provided data to inform and thereby enhance the counseling, but did not dictate precisely how the counseling should be performed. In the event that subjects did not immediately offer reasons for missed or off-time doses, clinicians were advised to say: "Let's talk more about any problems that you had last month." Most counseling sessions were completed within 10-15 min.

In the control arm, subjects continued to provide their EDM data to a study team member at their monthly visits, but these data remained blinded to both subjects and clinicians. However, control subjects whose monthly written self-reports indicated $<95 \%$ adherence were also flagged by a study team member for further counseling with a clinician. Thus, subjects in both groups whose adherence in the previous month appeared to be below 95\% were identified for counseling, with the difference being the flagging mechanism-EDM for intervention subjects and self-reported adherence for controls. In the counseling sessions with control subjects, which were guided by selfreported adherence, the standard of care in China, clinicians were similarly advised to inquire about recent problems that might have affected dose-taking.

To ensure that intervention procedures would be followed, members of the Boston-based team reviewed procedures on-site and conducted training with the local study team and clinicians prior to implementation of the intervention. The training focused on the set-up and provision of the EDM print-out to subjects and clinicians, which involved a 1/2-day period of overview and practice with the nurses and local study team, and the adherence counseling sessions. The latter included a $3-\mathrm{h}$ group training with all the clinicians at the DSPH clinic counseling that emphasized: (1) the goal of counseling was to help subjects to improve their medication-taking behavior, not to scold them about poor adherence; (2) the importance of discussing the EDM print-out with subjects; (3) posing practical questions to subjects on ways to improve adherence in the next month. Subsequent practice and role-playing were then conducted in two 3-h sessions.

\section{Measures}

Our primary outcome variable was adherence as measured by EDM. Specifically, we used the EDM adherence metric that was found to be most strongly associated with viral suppression (HIV RNA $<400$ copies/ml) in analysis of the pre-intervention data, EDM 'proportion taken within dose time' [40]. This measure estimated monthly adherence as the proportion of prescribed doses taken on time, e.g., within $1 \mathrm{~h}$ of scheduled dose time ([number of doses taken $\pm 1 \mathrm{~h}$ of dose time]/[total number of prescribed doses]). Dose times were selected by patients in consultation with their clinician, and could be changed if necessary. However, the two daily doses had to be spaced $12 \mathrm{~h}$ apart to optimize the pharmacokinetics of the drugs. Thus a patient was free to choose $9 \mathrm{am} / 9 \mathrm{pm}$ as his/her dose times, but could not choose 9 am/8:30 pm Any dose taken outside of this $\pm 1 \mathrm{~h}$ window was considered non-adherent for that dose. For more details, see Gill et al. 2009 [40].

Self-reported adherence, used to identify low adherers in the control group during the intervention period, was assessed from subjects' written responses to questions on the monthly form. These included: (1) a visual analog scale (VAS) of proportion of ART medications taken in the previous month; (2) a series of 6 yes/no questions about medication-taking behavior in the previous month (being careless, forgetting, stopping treatment due to feeling better, not taking medications while at work, taking pills early or late, sharing medications); and (3) two quantitative questions on the number of days medications were not taken and number of days medications were taken early or late. A subject in the control arm was flagged as a 'low adherer' if he/she reported $<95 \%$ on the VAS, answered 'yes' to any one of the six behavioral questions, or reported more than 0 days for either of the quantitative questions.

CD4 count and HIV viral load were tested at baseline, randomization (month 6), and post-intervention (month 12). CD4 counts were measured at a local laboratory by flow cytometry (FACSCalibur, Becton-Dickinson, San Carlos, CA, USA). We calculated change in CD4 
count for each subject by subtracting month $6 \mathrm{CD} 4$ from month 12 CD4 count. HIV viral loads were measured with an Organon Teknica NucliSens analyzer (BioMerieux, Boxtel, Netherlands), at the Center for Disease Control laboratory in Kunming, the provincial capital. The lower limit of the viral load assay was 400 copies per ml.

\section{Statistical Analysis}

In the assessment of the intervention, we included data for the 64 subjects who completed the study. Data were analyzed using SAS version 9.1 (SAS Institute, Cary, NC, USA). We compared mean adherence between the intervention and control groups at months 6 and 12, mean within-group adherence between the pre-intervention period and intervention period, CD4 count at months 6 and 12, and change in CD4 count from months 6 to 12 between groups using Student's $t$-tests. Cochran Mantel-Haenszel $(\mathrm{CMH}) \chi^{2}$ tests were used to compare proportions achieving 95\% adherence and proportions with HIV RNA $<400$ copies/ml at month 12. Demographic and socioeconomic characteristics of intervention and control subjects were compared using $t$-tests for continuous variables and $\mathrm{CMH}$ tests for categorical variables. Findings are presented as means with standard deviations (SD) or risk ratios (RR) and $95 \%$ confidence intervals (CI), with corresponding test statistics. All inferences were made based on a type I error rate equal to 0.05 . Each analysis was also adjusted for education, the only variable for which there was a significant difference between intervention and control subjects at randomization. Because effect size estimates did not change significantly, and due to our small sample size, we present only the unadjusted results.

Five subjects had months where data were missing due to EDM pill bottle malfunctions. In these cases, the previous month's adherence was used in calculations of single-month adherence (e.g., month 11 was used for month 12 adherence). For cumulative calculations (e.g., adherence during the pre-intervention or intervention period), the missing month's data were excluded and the cumulative indicator was calculated using 5 months of EDM data. Repeated openings within the same scheduled dose time were excluded.

\section{Results}

\section{Subject Flow}

Subject enrollment took place between June and November 2006. During this period, a total of 97 eligible patients attended the DSPH, all of whom were offered participation in the study. Of these, 80 were enrolled. The main reasons for non-participation were: (1) difficulty completing interviews due to language barriers (some clinic patients spoke a very heavy local dialect); (2) lack of availability to complete interviews; and (3) unwillingness to use the EDM pill bottle. Following 12 months of participation by each subject, data collection was completed by the end of November 2007. Of enrolled subjects, five died, and seven dropped out during the 6-month pre-intervention period (Fig. 1). The remaining 68 subjects were randomized into intervention and control arms, of which 64 completed the intervention period: 31 intervention subjects and 33 controls.

\section{Characteristics of Subjects}

Randomized subjects only differed in education background, with a higher proportion of intervention subjects having completed senior high or technical school (29 vs. $3 \% ; \mathrm{CMH}$ test statistic $=9.27 ; P$-value $=0.01)($ Table 1$)$. Fifty-one percent had been infected through IDU, although only $13 \%$ reported heroin use in the 3 months prior to randomization. Twenty-nine percent were treatment-naïve at baseline; mean duration on ART at month 6 was 10.9 months. Mean CD4 count was slightly higher among controls $(357$ vs. 297 cells $/ \mu \mathrm{l} ; t$-test statistic $=1.42 ; P$ value $=0.16$ ). The proportion of subjects with HIV RNA $<400$ copies/ml was $88 \%$ in each arm (CMH test statistic $=0.0082 ; P$-value $=0.93)$. The mean adherence of all randomized subjects was $85.7 \%$ during the pre-intervention period.

\section{Effect of EDM Feedback on Adherence}

We first compared adherence data at randomization (month 6) and month 12 (Table 2). At randomization, mean adherence among intervention and control subjects was comparable at 86.8 and $83.8 \%$, respectively ( $t$-test statistic $=-0.59 ; P$-value $=0.56)$. However, at month 12 , mean adherence among intervention subjects was much higher than among controls: 96.5 vs. $84.5 \%$ ( $t$-test statistic $=-3.20 ; P$-value $=0.003)$. Comparing within-arm changes between randomization and month 12 , mean adherence of intervention subjects increased (86.8-96.5\%, $t$-test statistic $=-2.84 ; P$-value $=0.008)$, with no significant change among controls $(83.8$ vs. $84.5 \%, t$-test statistic $=-0.14 ; \quad P$-value $=0.89)$. Similarly, contrasting mean adherence throughout the pre-intervention and intervention periods revealed a significant improvement in the intervention arm $(89.5$ vs. $96.4 \% ; t$-test statistic $=$ $-2.71 ; P$-value $=0.01)$, but no change in the control arm $(87.3$ vs. $84.1 \%, t$-test statistic $=0.68 ; P$-value $=0.50)$.

Point estimates for mean monthly adherence revealed significantly higher monthly adherence rates among the 
Table 1 Characteristics of subjects at randomization (6 months)

\begin{tabular}{|c|c|c|c|c|}
\hline Characteristic & Intervention $(n=34)$ & Control $(n=34)$ & Test statistic ${ }^{\mathrm{a}}$ & $P$-value \\
\hline Age (years) & $36.1(8.3)$ & $35.1(8.0)$ & -0.46 & 0.65 \\
\hline Male, $n(\%)$ & $25(74)$ & $25(74)$ & 0.00 & 1.000 \\
\hline Highest education level achieved, $n(\%)$ & & & 9.27 & 0.01 \\
\hline Primary school & $7(21)$ & $13(38)$ & & \\
\hline Middle school & $17(50)$ & $20(59)$ & & \\
\hline Secondary school & $10(29)$ & $1(3)$ & & \\
\hline Married, $n(\%)$ & $19(56)$ & $18(53)$ & 0.06 & 0.80 \\
\hline Han Ethnic background, $n(\%)$ & $14(41)$ & $17(50)$ & 0.53 & 0.47 \\
\hline Household size, persons (SD) & $4.2(1.5)$ & $4.5(1.4)$ & 1.13 & 0.26 \\
\hline Employed, $n(\%)$ & $10(31)$ & $12(38)$ & 0.27 & 0.60 \\
\hline Infected through injection drug use, $n(\%)$ & $18(53 \%)$ & $17(50)$ & 0.06 & 0.81 \\
\hline Used heroin in previous 3 months, $n(\%)$ & $4(12)$ & $5(15)$ & 0.13 & 0.72 \\
\hline Treatment-naïve at baseline (month 0 ), $n(\%)^{\mathrm{b}}$ & $9(26.5)$ & $10(29.4)$ & 0.07 & 0.79 \\
\hline Time on antiretroviral therapy, months (SD) & $10.5(4.3)$ & $11.3(6.6)$ & 0.59 & 0.56 \\
\hline CD4 count, mean cells/ $\mu \mathrm{l}(\mathrm{SD})$ & $297(145)$ & $357(196)$ & 1.42 & 0.16 \\
\hline HIV RNA <400 copies/ml (\%) & $30(88)$ & $28(88)$ & 0.008 & 0.93 \\
\hline Proportion with optimal adherence, $n(\%)^{\mathrm{c}}$ & & & 0.06 & 0.81 \\
\hline High $(\geq 95 \%)$ & $16(47)$ & $17(50)$ & & \\
\hline Low $(<95 \%)$ & $18(53)$ & $17(50)$ & & \\
\hline
\end{tabular}

${ }^{a}$ Test statistics are from Cochran Mantel-Haenszel $\chi^{2}$ tests for categorical variables and Student's $t$ tests for continuous variables

b Treatment-naive was defined as $\leq 2$ weeks duration on antiretroviral therapy

c Optimal adherence was defined as maintaining mean adherence $\geq 95 \%$ during months $1-5$ according to EDM and calculated as: \# prescribed doses taken $\pm 1 \mathrm{~h}$ prescribed dose time/prescribed doses

intervention arm than controls ( $P$-value $<0.001-.017$ for months $7,8,10-12 ; P$-value $=0.06$ for month 9 ), largely due to the sharp increase in adherence among previously low adherers (those with $<95 \%$ adherence before randomization). As shown in Fig. 2, mean monthly adherence in this intervention arm subgroup rose dramatically between months 6 and 8 and remained close to 95\% throughout the intervention period, whereas adherence among control arm low adherers did not change significantly.

In addition, EDM feedback improved the likelihood of achieving $95 \%$ adherence by month 12 and throughout the intervention period (see Table 2). Among intervention subjects, 26/31 (83.9\%) had $\geq 95 \%$ adherence in month 12 , compared to $13 / 33$ (39.4\%) among controls $(\mathrm{RR}=2.1$; $\mathrm{CI}$ : $1.4-3.3, \mathrm{CMH}$ test statistic $=13.08 ; P$-value $=0.0003)$. The effect of the intervention was more pronounced among low adherers, among whom 12/16 (75.0\%) intervention subjects, but only $2 / 17$ (11.8\%) controls achieved $\geq 95 \%$ adherence in month 12 (RR $=6.4$; CI: 1.7-24.2, $\mathrm{CMH}$ test statistic $=13.08 ; P$-value $=0.0003)$. Even those with high adherence prior to randomization appeared to benefit from EDM feedback, with 14/16 intervention (93.3\%) vs. $11 / 16$ control subjects $(68.8 \%)$ achieving $\geq 95 \%$ in month $12(\mathrm{RR}=1.4 ; \mathrm{CI}: 1.0-1.9, \mathrm{CMH}$ test statistic $=2.90$; $P$-value $=0.09)$.

\section{Effect of EDM Feedback on Markers} of HIV Progression

EDM feedback was not significantly associated with mean CD4 count at Month 12 (see Table 2). However, the proportion of subjects that experienced an increase in CD4 count was higher in the intervention arm (22/31 $(71.0 \%)$ vs. $15 / 31(48.4 \%)(\mathrm{CMH}$ test statistic $=3.23$; $P$-value $=0.07)$. More strikingly, the mean CD4 count rose by 90 cells/ $\mu$ l (SD 171.6) in intervention subjects during the intervention period, compared to a mean decline of 9 cells $/ \mu$ (SD 152.6) ( $t$-test statistic $=-2.4$; $P$-value $=0.02)$ among controls. The proportion of subjects with HIV RNA $<400$ copies/ml at month 12 was high in both arms, and did not differ significantly.

\section{Discussion}

This study has demonstrated that a simple intervention involving monthly adherence counseling based on EDM feedback significantly improved mean adherence among Chinese ART patients. CD4 counts were also significantly increased, though there was no effect on the degree of viral suppression at HIV RNA $<400$ copies/ml. In contrast to 
Table 2 Adherence and markers of HIV progression: intervention and control subjects

\begin{tabular}{|c|c|c|c|c|c|}
\hline Outcomes & $\begin{array}{l}\text { Intervention } \\
(N=31)\end{array}$ & $\begin{array}{l}\text { Control } \\
(N=33)\end{array}$ & Test statistic ${ }^{\mathrm{a}}$ & $P$-value & $\begin{array}{l}\text { Risk ratio } \\
(95 \% \mathrm{CI})\end{array}$ \\
\hline \multicolumn{6}{|l|}{ Adherence } \\
\hline \multicolumn{6}{|l|}{ Mean adherence, $\%(\mathrm{SD})^{\mathrm{b}}$} \\
\hline Month 6 & $86.8(18.5)$ & $83.8(21.8)$ & -0.59 & 0.5581 & - \\
\hline Month 12 & $96.5(4.8)$ & $84.5(21.0)$ & -3.20 & 0.0029 & - \\
\hline In pre-intervention period ${ }^{\mathrm{c}}$ & $89.5(13.8)$ & $87.3(16.7)$ & -0.55 & 0.5837 & - \\
\hline In intervention period ${ }^{c}$ & $96.4(3.4)$ & $84.1(21.4)$ & -3.24 & 0.0027 & - \\
\hline \multicolumn{6}{|l|}{ Proportion achieving $\geq 95 \%$ adherence, $n / N(\%)$} \\
\hline Month 12 & 26/31 (83.9) & 13/33 (39.4) & 13.08 & 0.0003 & $2.1(1.4-3.3)$ \\
\hline High $(\geq 95 \%)$ & $14 / 15(93.3)$ & $11 / 16(68.8)$ & 2.90 & 0.0885 & $1.4(1.0-1.9)$ \\
\hline Low $(<95 \%)$ & $12 / 16(75.0)$ & 2/17 (11.8) & 13.08 & 0.0003 & $6.4(1.7-24.2)$ \\
\hline In intervention period (mean) & 23/31 (74.2) & $11 / 33(33.3)$ & 10.55 & 0.0012 & $2.2(1.3-3.8)$ \\
\hline \multicolumn{6}{|l|}{ Markers of HIV progression } \\
\hline \multicolumn{6}{|l|}{ CD4 count } \\
\hline Month 12 , mean cells per $\mu \mathrm{l}$ (SD) & $401(256)$ & $357(157)$ & -0.83 & 0.4100 & \\
\hline \multicolumn{6}{|l|}{ Change in CD4 count, month 6 to month 12} \\
\hline Mean change in cells/ $\mu$ l (SD) & $90.0(171.6)$ & $-8.8(152.6)$ & -2.4 & 0.0197 & \\
\hline Proportion whose CD4 count rose, $n(\%)$ & $22(71.0)$ & $15(48.4)$ & -3.23 & 0.0722 & $1.5(1.0-2.2)$ \\
\hline HIV RNA <400 copies/ml, month $12, n(\%)$ & $27(87.1)$ & $31(93.9)$ & 0.87 & 0.3518 & $0.92(0.79-1.1)$ \\
\hline
\end{tabular}

a Test statistics are from Cochran Mantel-Haenszel $\chi^{2}$ tests for categorical variables and Student's $t$-tests for continuous variables. Student's $t$ tests for continuous variables except as noted

b Adherence measured via electronic drug monitor, calculated as: \# prescribed doses taken $\pm 1 \mathrm{~h}$ prescribed dose time/prescribed doses

c Pre-intervention period: months 1-6; Intervention period: months 7-12

Fig. 2 Monthly mean adherence among intervention and control subjects, stratified by mean adherence in preintervention period: high $(\geq 95 \%)$ vs. low $(<95 \%)$ adherers. Adherence measured via electronic drug monitor, calculated as: \# prescribed doses taken $\pm 1 \mathrm{~h}$ prescribed dose time/prescribed doses

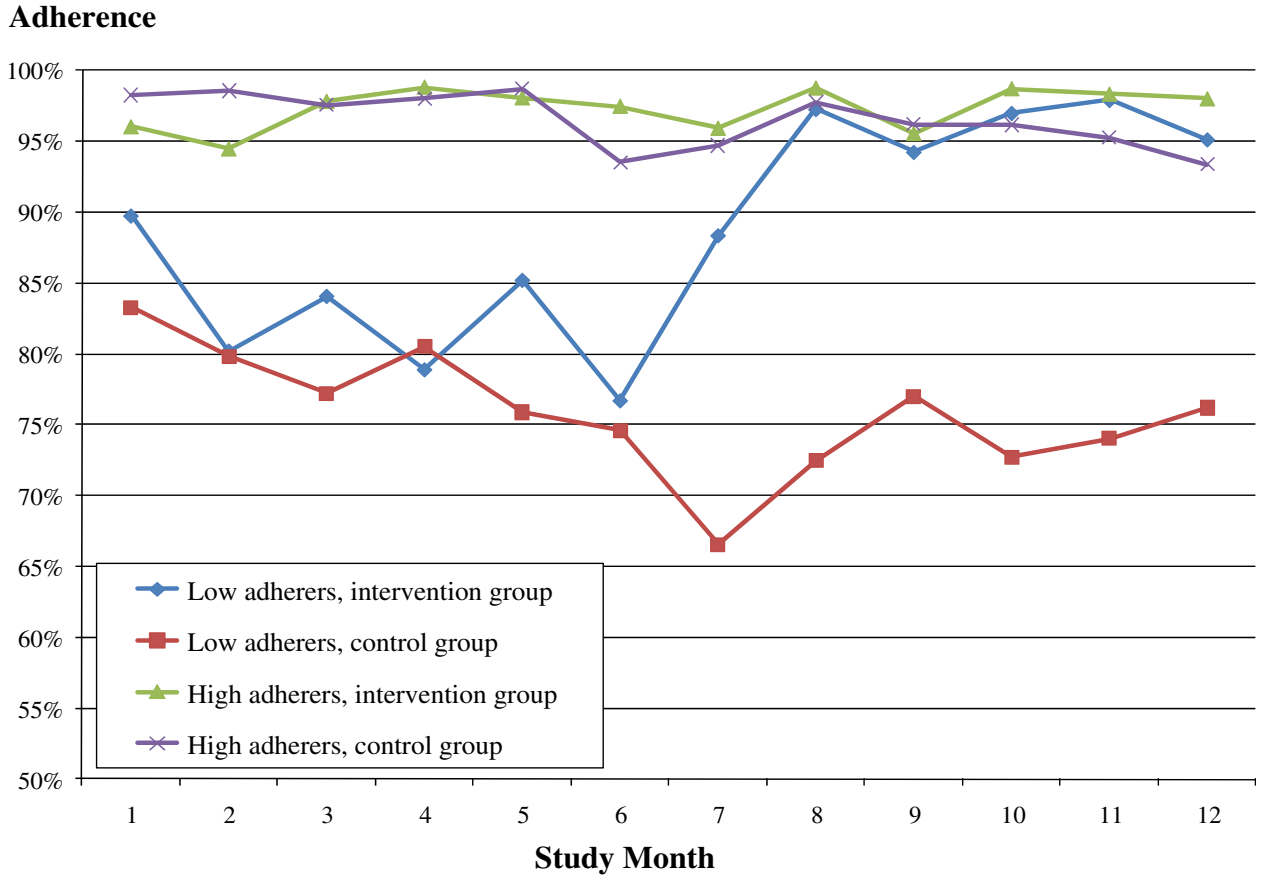

These findings add to our understanding of the potential effect of EDM feedback as an adherence intervention tool in several important ways. First, the effect on adherence was immediate-in the first 2 months-and occurred 
mainly among poorly adherent patients. This suggests that ART patients in China are capable of dramatic improvements in adherence, though the question of sustainability of effect remains unanswered. It is also consistent with a recent analysis which found that ART intervention studies that only enrolled participants with known or anticipated adherence issues were more likely to show a benefit [16]. In China, as in most developing countries, the enormous financial and public health ramifications of drug resistance and expensive second line regimens, in addition to increased mortality associated with poor ART adherence, argue strongly for focusing efforts to improve adherence among low adherers. That said, our findings suggest benefits to EDM feedback even among patients whose adherence is excellent; in our high adherence group, intervention subjects were less likely than controls to experience a decline in adherence to below $95 \%$ over time.

Relative to other ART adherence intervention studies that have utilized EDM feedback, AFL was simpler, involving provision of EDM-enhanced counseling only, without accompanying additional information, specific skills development, or financial incentives [24, 25]. This indicates that a focus on discussion of recent adherence behavior that relies on accurate information may be a particularly strong motivator for some patients, and is sufficient to produce meaningful behavior change. Intervention patients also maintained a significantly higher adherence level over a longer period ( 6 months) compared to other studies (3-4 months) [24, 25], suggesting the potential for sustained impact.

This study was not powered to detect a change in viral suppression and thus we were not surprised at the lack of such an effect. A number of other adherence intervention studies similarly successfully improved patients' adherence but did not observe significant changes in viral load measures [42, 43], while a recent meta-analysis of 18 ART adherence interventions also found an overall small but significant adherence effect but no improvement in viral suppression. In our study, high rates of pre-randomization viral suppression (88\%) made it unlikely that we could show significant changes in viral load parameters. This high rate of viral suppression in the context of mean adherence in the $85 \%$ range was probably the result of several factors, including the fact that all patients were on a NNRTI-based regimen that is more forgiving than protease inhibitor-based regimens when patients are less-than-optimally adherent [5].

An obvious question arises: why was EDM feedback so effective? Unfortunately, without detailed process data, we can only speculate about this, but several factors are likely salient, varying in importance for different patients. Some patients may be motivated by the ability to follow their progress, with no chance for self-delusion, much like individuals trying to lose weight may be encouraged by having to step on a scale periodically. Others may strongly desire to avoid enhanced counseling with a clinician, especially because EDM information precludes pretending that everything is okay, unlike self reports. This tendency may be reinforced by a particularly Chinese cultural tendency to highly respect clinicians. For those who struggle, the EDM feedback-based counseling provides an opportunity for meaningful discussion about medication-taking issues specific to the individual and points in time, which may provide certain patients just the focused discussion of behavior changes that they need. Its ultimate success depends on the counselor's approach, as well as on the patient's attitude and other variables that affect medicationtaking.

It is also important to recognize that the intervention included not just the counseling sessions, but a relatively reliable flagging system for the counseling (EDM data). Low adherers inclined to hide their poor adherence could do so if they were in the control group by self-reporting adherence above 95\%, unlike those in the intervention group. Our data on the number of intervention vs. control group subjects who were flagged for counseling sessionsconsistently higher in the former-support this hypothesis.

We also note a number of study limitations. First, the lack of a script for the EDM feedback counseling, while allowing clinicians flexibility, precluded collection and analysis of counseling process data. Further research is needed to understand better how EDM feedback alters the style and content of counseling sessions. Second, while the duration of the intervention ( 6 months) is one of the longest in the adherence literature, it is unclear how long EDM feedback must be continued to sustain the effect on adherence. Third, this study included no period of postintervention follow-up, and thus the long-term impact without the intervention is unknown and needs assessment. Lastly, we conducted the study in a distinctive cultural context-Chinese patients who were predominantly former IDUs. Whether EDM feedback would be as effective in other cultural contexts, or even in China in non-former IDU populations, remains to be tested.

Our results also raise several operational questions. First, does EDM feedback have a persistent effect after discontinuation, or must it be continued indefinitely? Second, while we used clinicians (both physicians and nurses) for the adherence counseling, it would be helpful to determine whether EDM feedback is effective if counseling is done by peers or family members. Third, how easily can the technical requirements of EDM feedback (computers, pill bottles, staff training) be adapted to other settings and at larger scales? We found that EDM feedback required a moderate degree of training. The basics of EDM use required a full day of training and practice with local 
staff; the intervention-related flagging of low adherers and counseling required another 1-1.5 days. After the first month or so of actual implementation, both staff and patients were comfortable with the technical details of EDM use and sustaining the intervention, once begun, was not burdensome. This is relevant since there is a prevailing sentiment in the literature that EDM are too complex and technically demanding to be of broad usefulness.

The results of this study show that adherence counseling utilizing EDM feedback was effective at achieving sustained rates of optimal adherence and that these behavioral changes translated into improved CD4 counts, an important marker of HIV progression. We conclude that this is a promising approach to improving adherence, with potential for wide applicability in other HIV populations and also possibly in the management of other chronic diseases. Given the distinctive context in which we conducted the study, further evaluation in diverse populations and settings is warranted.

Acknowledgments We thank Mary Jordan, Billy Pick, David Stanton, and Lois Bradshaw at USAID and Jonathan Simon, Donald Thea, and Deirdre Pierotti at Boston University. We also warmly acknowledge the contributions of our Boston-based program managers Mini Singh and Anna Knapp and our China-based field managers Wanju Wu, Matt Bobo, Guo Jinhua, James Chen, Ahmar Hashmi, and Jordan Tuchman. We are grateful for the support of Cheng Feng at Family Health International, Ray Yip at the Gates Foundation, and Connie Osborne and her colleagues at the WHOBeijing office.

Sources of Support This study was supported by a cooperative agreement (GHS-A-00-03-00030-00) between Boston University and the Office of Health and Nutrition of the United States Agency for International Development (USAID), with additional support from the World Health Organization (WHO) and the United States Centers for Disease Control. Dr. Gill's work was supported by NIH/NIAID K23 AI 62208. Dr. Wilson was supported for this work by Mid-Career Mentoring Award (K24 RR020300).

Open Access This article is distributed under the terms of the Creative Commons Attribution Noncommercial License which permits any noncommercial use, distribution, and reproduction in any medium, provided the original author(s) and source are credited.

\section{References}

1. Bangsberg DR, Hecht FM, Charlebois ED, Zolopa AR, Holodniy M, Sheiner L, et al. Adherence to protease inhibitors, HIV-1 viral load, and development of drug resistance in an indigent population. AIDS. 2000;14(4):357-66.

2. Gross R, Bilker WB, Friedman HM, Strom BL. Effect of adherence to newly initiated antiretroviral therapy on plasma viral load. AIDS. 2001;15(16):2109-17.

3. Hogg RS, Heath K, Bangsberg D, Yip B, Press N, O'Shaughnessy MV, et al. Intermittent use of triple-combination therapy is predictive of mortality at baseline and after 1 year of follow-up. AIDS. 2002;16(7):1051-8.
4. Paterson DL, Swindells S, Mohr J, Brester M, Vergis EN, Squier $\mathrm{C}$, et al. Adherence to protease inhibitor therapy and outcomes in patients with HIV infection. Ann Intern Med. 2000;133(1):21-30.

5. Bangsberg DR. Less than $95 \%$ adherence to nonnucleoside reverse-transcriptase inhibitor therapy can lead to viral suppression. Clin Infect Dis. 2006;43(7):939-41.

6. Palombi L, Marazzi MC, Guidotti G, Germano P, Buonomo E, Scarcella $\mathrm{P}$, et al. Incidence and predictors of death, retention, and switch to second-line regimens in antiretroviral-treated patients in Sub-Saharan African sites with comprehensive monitoring availability. Clin Infect Dis. 2008;48:115-22.

7. Simoni JM, Pearson CR, Pantalone DW, Marks G, Crepaz N. Efficacy of interventions in improving highly active antiretroviral therapy adherence and HIV-1 RNA viral load. A meta-analytic review of randomized controlled trials. J Acquir Immune Defic Syndr. 2006;43(Suppl 1):S23-35.

8. Detels R, Tarwater P, Phair JP, Margolick J, Riddler SA, Munoz A. Effectiveness of potent antiretroviral therapies on the incidence of opportunistic infections before and after AIDS diagnosis. AIDS. 2001;15(3):347-55.

9. Keiser P, Nassar N, Kvanli MB, Turner D, Smith JW, Skiest D. Long-term impact of highly active antiretroviral therapy on HIVrelated health care costs. J Acquir Immune Defic Syndr. 2001; 27(1):14-9.

10. Sendi PP, Bucher HC, Harr T, Craig BA, Schwietert M, Pfluger $\mathrm{D}$, et al. Cost effectiveness of highly active antiretroviral therapy in HIV-infected patients. Swiss HIV cohort study. AIDS. 1999;13(9):1115-22.

11. Valenti WM. Treatment adherence improves outcomes and manages costs. AIDS Read. 2001;11(2):77-80.

12. Kozal MJ, Hullsiek KH, Macarthur RD, Berg-Wolf M, Peng G, Xiang $\mathrm{Y}$, et al. The incidence of HIV drug resistance and its impact on progression of HIV disease among antiretroviral-naive participants started on three different antiretroviral therapy strategies. HIV Clin Trials. 2007;8(6):357-70.

13. Hogg RS, Bangsberg DR, Lima VD, Alexander C, Bonner S, Yip $\mathrm{B}$, et al. Emergence of drug resistance is associated with an increased risk of death among patients first starting HAART. PLoS Med. 2006;3(9):e356.

14. Colson P, Ravaux I, Yahi N, Tourres C, Gallais H, Tamalet C. Transmission of HIV-1 variants resistant to the three classes of antiretroviral agents: implications for HIV therapy in primary infection. AIDS. 2002;16(3):507-9.

15. Siegrist CA, Yerly S, Kaiser L, Wyler CA, Perrin L. Mother to child transmission of zidovudine-resistant HIV-1. Lancet. 1994;344(8939-8940):1771-2.

16. Amico KR, Harman JJ, Johnson BT. Efficacy of antiretroviral therapy adherence interventions: a research synthesis of trials, 1996 to 2004. J Acquir Immune Defic Syndr. 2006;41(3):285-97.

17. DiIorio C, Resnicow K, McDonnell M, Soet J, McCarty F, Yeager K. Using motivational interviewing to promote adherence to antiretroviral medications: a pilot study. J Assoc Nurses AIDS Care. 2003;14(2):52-62.

18. Goujard C, Bernard N, Sohier N, Peyramond D, Lancon F, Chwalow $\mathrm{J}$, et al. Impact of a patient education program on adherence to HIV medication: a randomized clinical trial. J Acquir Immune Defic Syndr. 2003;34(2):191-4.

19. Parsons JT, Golub SA, Rosof E, Holder C. Motivational interviewing and cognitive-behavioral intervention to improve HIV medication adherence among hazardous drinkers: a randomized controlled trial. J Acquir Immune Defic Syndr. 2007;46(4):44350.

20. Safren SA, Otto MW, Worth JL, Salomon E, Johnson W, Mayer $\mathrm{K}$, et al. Two strategies to increase adherence to HIV antiretroviral medication: life-steps and medication monitoring. Behav Res Ther. 2001;39(10):1151-62. 
21. Bangsberg DR. Monitoring adherence to HIV antiretroviral therapy in routine clinical practice: the past, the present, and the future. AIDS Behav. 2006;10(3):249-51.

22. Bangsberg DR. Preventing HIV antiretroviral resistance through better monitoring of treatment adherence. J Infect Dis. 2008;197(Suppl 3):S272-8.

23. Arnsten JH, Demas PA, Farzadegan H, Grant RW, Gourevitch $\mathrm{MN}$, Chang CJ, et al. Antiretroviral therapy adherence and viral suppression in HIV-infected drug users: comparison of self-report and electronic monitoring. Clin Infect Dis. 2001;33(8):1417-23.

24. Smith SR, Rublein JC, Marcus C, Brock TP, Chesney MA. A medication self-management program to improve adherence to HIV therapy regimens. Patient Educ Couns. 2003;50(2):187-99.

25. Rosen MI, Dieckhaus K, McMahon TJ, Valdes B, Petry NM, Cramer $\mathbf{J}$, et al. Improved adherence with contingency management. AIDS Patient Care STDS. 2007;21(1):30-40.

26. World Health Organization. The world health report-working together for health. Geneva: World Health Organization; 2006.

27. State Council AIDS Working Committee Office \& UN Theme Group on AIDS in China. A joint assessment of HIV/AIDS prevention, treatment and care in China (2007). Beijing: December 1, 2007.

28. UNAIDS. Report on the global AIDS epidemic 08. Mexico City: Joint United Nations Programme on HIV/AIDS; August 2008.

29. Wu Z, Rou K, Detels R. Prevalence of HIV infection among former commercial plasma donors in rural eastern China. Health Policy Plan. 2001;16(1):41-6.

30. Ministry of Health of China, UNAIDS, WHO. 2005 update on the HIV/AIDS epidemic and response in China. Beijing: Ministry of Health; 2006.

31. Yan J, Zheng X, Zhang X, et al. The survey of prevalence of HIV infection among paid blood donors in one county in China. Zhonghua Liu Xing Bing Xue Za Zhi [Chinese Journal of Infectious Diseases]. 2000;21(1):10-3.

32. He N, Detels R. The HIV epidemic in China: history, response, and challenge. Cell Res. 2005;15(11-12):825-32.

33. Wu Z, Sullivan SG, Wang Y, Rotheram-Borus MJ, Detels R. Evolution of China's response to HIV/AIDS. Lancet. 2007;369 (9562):679-90.
34. Zhang F, Hsu M, Yu L, Wen Y, Two J, Zhang R. Initiation of the national ARV therapy program in rural China. Beijing: July 2004.

35. Zhang F, Hsu M, Yu L, Wen Y, Pan J. Initiation of the national free antiretroviral therapy program in rural China. In: Kaufman J, Kleinman A, Saich T, editors. AIDS and social policy in China. Cambridge, MA: Harvard University Asia Center; 2006. p. 96104.

36. Starks H, Simoni J, Zhao H, Huang B, Fredriksen-Goldsen K, Pearson $\mathrm{C}$, et al. Conceptualizing antiretroviral adherence in Beijing, China. AIDS Care. 2008;20(6):607-14.

37. Sabin LL, Desilva MB, Hamer DH, Keyi X, Yue Y, Wen F, et al. Barriers to adherence to antiretroviral medications among patients living with HIV in southern China: a qualitative study. AIDS Care. 2008;20(10):1242-50.

38. Wang H, He G, Li X, Yang A, Chen X, Fennie KP, et al. Selfreported adherence to antiretroviral treatment among HIVinfected people in Central China. AIDS Patient Care STDS. 2008;22(1):71-80.

39. Fisher JD, Fisher WA, Amico KR, Harman JJ. An informationmotivation-behavioral skills model of adherence to antiretroviral therapy. Health Psychol. 2006;25(4):462-73.

40. Gill CJ, Sabin LL, Hamer DH, Xu K, Zhang J, Li T, Wu W-J, Wilson IB, Bachman DeSilva M. Importance of dose timing to achieving undetectable viral loads. AIDS Behav. 2009; Published online: April 8, 2009.

41. Xiao Y, Kristensen S, Sun J, Lu L, Vermund SH. Expansion of HIV/AIDS in China: lessons from Yunnan Province. Soc Sci Med. 2007;64(3):665-75.

42. Remien RH, Stirratt MJ, Dolezal C, Dognin JS, Wagner GJ, Carballo-Dieguez A, et al. Couple-focused support to improve HIV medication adherence: a randomized controlled trial. AIDS. 2005;19(8):807-14.

43. Margolin A, Avants SK, Warburton LA, Hawkins KA, Shi J. A randomized clinical trial of a manual-guided risk reduction intervention for HIV-positive injection drug users. Health Psychol. 2003;22(2):223-8. 